\title{
Edema na hanseníase: aspectos clínicos e terapêuticos
}

\author{
Edema in leprosy: clinical and therapeutical aspects
}

\author{
Attilio Valentini, José A.C. Nery, Ana Maria Salles, \\ Leila M.M. Vieira e Euzenir N. Sarno
}

\begin{abstract}
Resumo Durante o curso da hanseníase, o edema comumente descrito como um sintoma de estados reacionais, pode ocorrer. Tanto o diagnóstico como a terapêutica adequada são, freqüentemente, difíceis de conseguir e assim podem acarretar permanente dano aos membros inferiores. Em um ano de acompanhamento, pacientes hansenianos - 10 multibacilares e 1 paucibacilar -, que foram submetidos a um protocolo clínico para o diagnóstico e classificação histopatológica, apresentaram clinicamente edema, localizado ou sistêmico. Entre estes pacientes, cinco apresentaram simultaneamente outros sintomas de reação, 4 foram classificados como reação Tipo I e um como reação tipo II. Por outro lado, três pacientes não apresentaram reação no momento do diagnóstico, mas desenvolveram alguns aspectos de reação posteriormente (2 tiveram neurite e um teve reação tipo I). Os edemas observados precedendo ou associados a quadros reacionais apresentaram ótima resposta clínica às drogas de ação anti-inflamatória (corticóide, talidomida e pentoxifilina) utilizadas para o tratamento dos estados reacionais, na ausência de qualquer outro tratamento normalmente usado para edema. Embora necessitem ser confirmados por estudos controlados, estes dados sugerem fortemente que mecanismos imunológicos estejam envolvidos na fisiopatologia dos edemas na hanseníase.
\end{abstract}

Palavras-chaves: Edema. Hanseníase. Reação. Talidomida. Corticóide. Pentoxifilina. TNF. IL-1.

\begin{abstract}
Edema, which is commonly described as a symptom of reactional states, may occur during the course of leprosy. Both diagnosis and adequate treatment measures are often difficult to achieve and failure to do so may result in permanent damage to the lower limbs. In a one-year follow-up study of leprosy patients - ten multibacillary and one paucibacillary who had been submitted to a clinical protocol for diagnosis and pathological classification, a clinical pattern of localized and/or systemic edema was observed. Among these patients, five simultaneously presented other symptoms related to reactional states, 4 were diagnosed as Type I, and one as Type II. On the other hand, while three of the patients did not present reaction at the time when edema was diagnosed, they did develop some aspects of reactional disease later on (two had neuritis e one had Type I reaction). The edemas that preceded or were associated with reactional episodes showed clinical regression as a result of specific treatment against reactions (corticosteroids and/or pentoxifylline and/or thalidomide) in the absence of another treatment normally used for edemas. Although these data need to be confirmed by controlled studies, they strongly suggest that immunological mechanisms are involved in the physiopathology of edema in leprosy.
\end{abstract}

Key-words: Edema. Leprosy. Reaction. Thalidomide. Corticosteroids. Pentoxifylline. TNF. IL-1.

\footnotetext{
Laboratório de Hanseníase, Instituto Oswaldo Cruz, Fundação Oswaldo Cruz, Rio de Janeiro; Disciplina de Dermatologia, Departamento de Clínica Médica, Universidade Federal Fluminense, Niterói; Disciplina de Patologia Geral, Departamento de Patologia e Laboratórios, Universidade do Estado do Rio de Janeiro, Rio de Janeiro, RJ, Brasil.

Parcialmente financiado pela Organização Mundial de Saúde (OMS), pelo Conselho Nacional de Pesquisa (CNPq) e Fundação de Amparo à Pesquisa do Rio de Janeiro (FAPERJ).

Endereço para correspondência: Dra. Euzenir N. Sarno. Laboratório de Hanseníase/FIOCRUZ, Av. Brasil 4365, Manguinhos, 21045-900 Rio de Janeiro, RJ, Brasil. Telefax: 5521 270-9997.

Recebido para publicação em 29/3/98.
} 
A ocorrência de edema no curso da hanseníase, apesar de ser de relativa freqüência passa, muitas vezes, despercebida na rotina diagnóstica. Na hanseníase virchowiana o edema de pernas e tornozelos pode preceder em meses ou anos as lesões cutâneas clássicas, sendo comumente bilateral e vespertino?

Classicamente, os edemas são descritos no curso de estados reacionais. Na reação tipo I (reação reversa) pode ocorrer edema nos lábios, simulando o edema angioneurótico ou envolvendo a região palpebral, com instalação súbita de um dia para outro1, assim como edema, de discreto a marcante de pés, mãos e face 9 . O edema oral ou facial na reação tipo I acomete principalmente pacientes com formas clínicas borderlines, especialmente borderlinelepromatoso $B L 6$. Na reação tipo II, pode ocorrer edema de extremidades independente da magnitude das lesões cutâneas 11 . Eventualmente, podem surgir formas generalizadas de edema, simulando quadros anasarca-like.

O edema nas diferentes formas clínicas da hanseníase assim como no curso dos estados reacionais foi revisto por Wheate ${ }^{23}$. Este autor relatou que, independente da forma clínica, o edema de mãos e pés se associava ao espessamento e alteração de sensibilidade dos principais ramos nervosos (principalmente ulnar e fibular), devendo ser considerado como uma condição reacional e, tratado como tal.

A presença de edema nos pacientes com hanseníase é um fator de dificuldade no manuseio terapêutico. A cicatrização de úlceras nos pés é prejudicada e nos casos de pacientes com edema crônico, contraturas deformantes de mãos e pés não são infreqüentes.

O edema na hanseníase representa uma complicação pela limitação funcional imposta e pelas alterações orgânicas, muitas vezes sistêmicas, necessitando uma abordagem terapêutica adequada. Apesar disto, poucos ainda são os estudos relacionados ao esclarecimento dos mecanismos patogênicos que permitam uma melhor caracterização do mesmo.

Para melhor caracterizar clinicamente estes edemas no curso da hanseníase e assim avaliar os possíveis mecanismos fisiopatogênicos associados foram estudados pacientes hansenianos que apresentavam ou vieram apresentar edema no curso do acompanhamento.

\section{MATERIAL E MÉTODOS}

Foram selecionados 11 pacientes com hanseníase, que durante o acompanhamento no Ambulatório Souza Araújo - Laboratório de Hanseníase da Fundação Oswaldo Cruz, FIOCRUZ/RJ no período de setembro/1996 a setembro/1997, apresentaram quadro clínico de edema.

Os pacientes foram classificados de acordo com os critérios de Ridley e Jopling12 em: BT (Borderline-tuberculóide), BB (borderlineborderline), BL (borderline-lepromatoso) e LL (lepromatoso-lepromatoso). Todos os pacientes multibacilares foram submetidos ao esquema de poliquimioterapia (PQT/MB) recomendado pela Organização Mundial de Saúde (OMS) e pela Coordenação Nacional de Dermatologia Sanitária do Ministério da Saúde (CNDS/MS), recebendo doses mensais supervisionadas de $600 \mathrm{mg}$ de rifampicina e $300 \mathrm{mg}$ de clofazimina e doses diárias autoadministradas de $100 \mathrm{mg}$ de dapsona e $50 \mathrm{mg}$ de clofazimina durante um período de 24 meses. Os pacientes paucibacilares foram submetidos ao esquema de poliquimioterapia (PQT/PB), recebendo doses mensais supervisionadas de $600 \mathrm{mg}$ de rifampicina e $300 \mathrm{mg}$ de clofazimina e doses diárias autoadministradas de $100 \mathrm{mg}$ de dapsona durante um período de 6 meses.

Os pacientes foram submetidos à avaliação laboratorial através de exames de bioquímica (glicose, eletrólitos, hepatograma, proteína total e frações), hemograma completo, VHS e urinálise, a fim de se avaliar eventuais danos e/ou patologias que pudessem ser associados ao edema.

Nenhum dos dos pacientes apresentava ou veio a apresentar adenomegalias associadas às áreas com edema.

Após cuidadoso exame clínico e avaliação dos exames laboratoriais foram excluídos os pacientes que apresentaram alterações ou patologias associadas (cardíacas, renais, hepáticas, vasculares, nutricionais) que justificassem a ocorrência de edema.

Os edemas foram caracterizados, segundo as seguintes variáveis: localização: forma localizada (envolvimento das mãos e/ou pés), forma regional (envolvimento de braços e/ou pernas) e forma mista (envolvimento simultâneo localizado e regional); simetria: (envolvimento uni ou bilateral, avaliando-se a relação entre as 
partes); tempo de evolução (período relatado da instalação do edema); consistência (depressível ou não quanto a digito pressão); dor (presença ou ausência a digito pressão); cor (eventual alteração da coloração cutânea na área com edema); temperatura (eventual alteração desta na área com edema); queixa neural associada (relato subjetivo do paciente de hiperestesia, parestesia ou anestesia na área com edema, sem relação ou exacerbação à palpação do trajeto nervoso correspondente); resposta ao teste da histamina ou teste da pilocarpina, realizado na área com edema, para se avaliar eventuais alterações no sistema nervoso autônomo, que pudessem contribuir na formação do edema. O teste de histamina não foi realizado nos pacientes melanodérmicos (e sim o teste da pilocarpina) para evitar dificuldades quanto a leitura do mesmo.

Exame bacteriológico para determinação do índice baciloscópico foi realizado em linfa colhida de 6 locais, conforme CNDS. O índice baciloscópico inicial variou de 0,16 a 3,66.

Os quadros clínicos de reação tipo I (reação reversa), reação tipo II e neurite isolada foram baseados em critérios clínicos, descritos a seguir:

Reação reversa (reação tipo I): reativação de lesões pré-existentes, tipo placa, com sinais de inflamação aguda, tais como eritema, edema e hipersensibilidade; aparecimento de lesões novas tipo placa, com características semelhantes às anteriores; espessamento de nervos periféricos, acompanhados de dor espontânea ou à compressão; distúrbios sistêmicos como, por exemplo, mal estar geral e, eventualmente, febre.

Reação tipo II (eritema nodoso hansênico): aparecimento de nódulos dérmicos ou subcutâneos eritematosos, quentes, móveis, por vezes dolorosos; lesões eritematosas com formação de vesículas, bolhas, evoluindo muitas vezes para ulcerações; sintomas sistêmicos tais como: febre, adenomegalia, perda de peso, artralgia, mialgia, etc; espessamento, dor e sensibilidade de nervos.

Neurite isolada: aparecimento de dor espontânea ou à compressão em nervos periféricos, acompanhada ou não, de edema localizado. Para efeito de classificação denominou-se neurite isolada aqueles casos que, durante todo o episódio reacional apresentaram apenas sintomas neurais sem manifestações cutâneas de reação tipo I e tipo II.

Os pacientes foram ainda acompanhados clinicamente, quanto à evolução do edema e o curso da hanseníase, para melhor avaliar sua representação clínica no espectro da hanseníase.

No momento do episódio reacional, os pacientes receberam a terapêutica adequada, ou seja, para o tratamento de reação tipo I, ou neurite isolada ou ainda, mulheres com reação tipo II em idade fértil, a droga de escolha foi a prednisona na dosagem de 1 a $2 \mathrm{mg} / \mathrm{kg} / \mathrm{dia}$, de acordo com avaliação clínica. Para tratamento da reação tipo II a droga de escolha foi a talidomida, exceto quando houve contraindicação (mulheres em idade fértil), na dose de $100-400 \mathrm{mg} /$ dia conforme a gravidade do quadro.

Quanto à evolução clínica, os pacientes foram subdivididos em 2 grupos:

Grupo A: pacientes que no momento da seleção apresentavam edema concomitante a outros aspectos clínicos da reação.

Grupo B: pacientes que no momento da seleção apresentavam apenas edema.

\section{RESULTADOS}

Entre os 11 pacientes analisados (Tabela 1), observou-se um predomínio de formas multibacilares (4 LL, $3 \mathrm{BB}, 3 \mathrm{BL}, 1 \mathrm{BT}$ ). Quanto ao sexo, 10 eram do sexo masculino e 1 do sexo feminino. A idade variou entre 18 e 65 anos (média $=42 \pm 16$ ).

O momento terapêutico dos pacientes foi diverso: 6 estavam em alta pós-PQT (do $2^{\circ}$ ao $82^{\circ}$ mês, com IB de 0 a 3,66), 4 na vigência de PQT/MB (do 6은 $24^{\circ}$ mês, com IB de 0 a 3,33 ) e 1 paciente ainda não iniciara medicação específica (IB $=1+$ ) (Tabela 1$)$.
Cinco pacientes apresentavam edema no momento da seleção associado a reações hansênicas (grupo A: 4 em reação tipo I e 1 reação tipo II), estando 4 pacientes na vigência

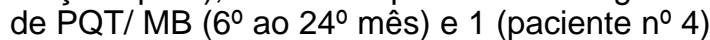
ainda virgem de tratamento. Interessante ressaltar que destes, 3 pacientes (no 1, 4 e 5) já apresentavam edema previamente (num período variável de 1 a 6 meses) à instalação dos quadros reacionais (reações tipo I).

Os demais seis pacientes (Tabela 2) apresentavam edema, no momento da seleção, 
Tabela 1 - Distribuição dos pacientes quanto a sexo, cor, idade, forma clínica, momento clínico e momento terapêutico.

\begin{tabular}{|c|c|c|c|c|c|c|c|}
\hline $\begin{array}{c}\text { Pacientes } \\
n^{0}\end{array}$ & Sexo & Cor & Idade & Forma clínica & IB & Momento clínico & Momento terapêutico (PQT) \\
\hline 1 & $\mathrm{M}$ & pta & 65 & $\mathrm{BL}$ & $3+$ & reação tipo I & $13^{\circ}$ mês \\
\hline 2 & $\mathrm{~F}$ & pta & 42 & LL & $3+$ & reação tipo II & $24^{\circ}$ mês \\
\hline 3 & $\mathrm{M}$ & pta & 25 & BB & $3,33+$ & reação tipo I & 6ํmês \\
\hline 4 & M & bca & 18 & BB & $1+$ & reação tipo I & virgem de tratamento \\
\hline 5 & M & pta & 57 & BB & 0 & reação tipo I & $12^{\circ}$ mês \\
\hline 6 & $\mathrm{M}$ & bca & 31 & BT & 0 & sem reação & $2^{2}$ mês pós-alta PQT* \\
\hline 7 & $\mathrm{M}$ & bca & 62 & $B L$ & $0,66+$ & sem reação & 7º mês pós-alta PQT \\
\hline 8 & $\mathrm{M}$ & bca & 46 & LL & $0,16+$ & sem reação & $82^{\circ}$ mês pós-alta PQT \\
\hline 9 & $\mathrm{M}$ & pda & 56 & LL & $3,66+$ & sem reação & 9º mês pós-alta PQT \\
\hline 10 & $\mathrm{M}$ & pta & 31 & LL & $0,33+$ & sem reação & 16º mês pós-alta PQT \\
\hline 11 & M & bca & 29 & $B L$ & $0,16+$ & sem reação & $60^{\circ}$ mês pós-alta* \\
\hline
\end{tabular}

IB = índice baciloscópico; $M=$ masculino; F = feminino; * PQT/PB.

Tabela 2 - Evolução clínica dos pacientes do Grupo B.

\begin{tabular}{|c|c|c|c|c|c|c|c|}
\hline $\begin{array}{c}\text { Pacientes } \\
\text { no }\end{array}$ & $\begin{array}{c}\text { Tempo de } \\
\text { evolução do } \\
\text { edema }\end{array}$ & $\begin{array}{c}\text { Queixa } \\
\text { neural } \\
\text { associada }\end{array}$ & $\begin{array}{l}\text { Evolução } \\
\text { clínica } \\
\text { (mês) }\end{array}$ & $\begin{array}{c}\text { Terapia } \\
\text { vigente* }\end{array}$ & $\begin{array}{c}\text { Terapia } \\
\text { associada** }^{*}\end{array}$ & $\begin{array}{l}\text { Resposta } \\
\text { clínica }\end{array}$ & $\begin{array}{c}\text { Tempo da } \\
\text { resposta } \\
\text { clínica }\end{array}$ \\
\hline 6 & $6 \mathrm{~m}$ & hiperestesia & $\begin{array}{l}\text { neurite } \\
\text { (1 mês após) }\end{array}$ & não & pred (30mg) & $\begin{array}{l}\text { remissão da neurite } \\
\text { e melhora do edema }\end{array}$ & $1 \mathrm{~m}$ \\
\hline 7 & $1 \mathrm{~m}$ & parestesia & sem reação & $\mathrm{AMT} \star 2$ & $\begin{array}{l}\text { não } \\
\text { (50mg) }\end{array}$ & $\begin{array}{l}\text { melhora da queixa } \\
\text { neural e do edema }\end{array}$ & 2 sem \\
\hline 8 & $3 \mathrm{~m}$ & A & sem reação & $\begin{array}{l}\mathrm{PTX} * 3 \\
(400 \mathrm{mg})\end{array}$ & $\begin{array}{l}\text { betam.*4 } \\
(1 \mathrm{mg})\end{array}$ & $\begin{array}{l}\text { remissão do } \\
\text { edema }\end{array}$ & 1 sem \\
\hline 9 & $11 / 2 \mathrm{~m}$ & hiperestesia & sem reação & não & $\begin{array}{l}\text { pred } \\
(20 \mathrm{mg})\end{array}$ & remissão do edema & 1 sem \\
\hline 10 & 1 sem & parestesia & $\begin{array}{l}\text { reação tipo I } \\
\text { (3 meses após) }\end{array}$ & $\begin{array}{l}\mathrm{PTX} * 3 \\
(400 \mathrm{mg})\end{array}$ & $\begin{array}{l}\text { pred } \\
\text { (60mg) }\end{array}$ & remissão do edema & 1 sem \\
\hline 11 & $1 \mathrm{~m}$ & $A$ & $\begin{array}{l}\text { neurite } \\
\text { (1 mes após) }\end{array}$ & não & $\begin{array}{l}\text { pred } \\
(60 \mathrm{mg})\end{array}$ & $\begin{array}{l}\text { remissão da neurite } \\
\text { e do edema }\end{array}$ & 3 sem \\
\hline
\end{tabular}

*Terapia vigente- medicamentos em uso no momento da seleção, para o tratamento de episódios reacionais.

** Terapia associada- medicamentos adicionados em função de agravamento de episódios reacionais.

${ }_{2} \mathrm{AMT}=$ amitriptilina. Uso devido à queixa de parestesia (queixa subjetiva sem características objetivas de neurite).

${ }^{*}$ PTX = pentoxifilina em uso como tentativa de abordagem terapêutica do edema.

${ }^{*} 4$ betam = betametasona. Automedicação

$\mathrm{A}=$ ausente

sem estarem em curso de estados reacionais (grupo B), estando todos em alta terapêutica pós-PQT (5 PQT/ MB e $1 \mathrm{PQT} / \mathrm{PB}$ ).

Os edemas localizaram-se em 7 pacientes apenas nos membros inferiores (Tabela 3): 5 pacientes de forma regional, nas pernas (4 de forma assimétrica - nos 3, 8, 10 e 11; e 2 simétrica - nos 5 e 9), 3 pacientes (nos 1, 2 e 4) com acometimento misto (edema nos pés e/ou pernas, junto a edema de braços e/ou mãos), 1 paciente (no 6) de forma localizada, assimétrica no pé esquerdo. Os membros superiores estiveram envolvidos em 4 pacientes: 1 paciente ( $n$ 을 de forma localizada e assimétrica e 3 pacientes (nos 1, 2 e 4) com acometimento misto (edema nos pés e/ou pernas, junto ao edema de braços e/ou mãos).

O tempo de evolução dos edemas variou de 4 dias a 6 meses (Tabela 3).

Todos os edemas foram depressíveis, sendo dolorosos a digito pressão em 2 casos (nos 6 e 9). Houve alteração da temperatura local (hipertermia) em 2 pacientes (nos 3 e 9).

A cor da pele na área dos edemas (Tabela 3) estava alterada em 7 dos pacientes ( 3 cianótica, 3 eritematosa e 1 eritematocianótica).

A associação de queixa neural (Tabela 3 ) na área dos edemas esteve presente em 5 pacientes (3 com parestesia e 2 com hiperestesia). 
Tabela 3 - Distribuição e caracterização dos edemas*.

\begin{tabular}{|c|c|c|c|c|c|c|c|c|}
\hline $\begin{array}{c}\text { Pacientes } \\
\text { no }\end{array}$ & Localização & $\begin{array}{c}\text { Tempo } \\
\text { de } \\
\text { evolução**. }^{\text {To }}\end{array}$ & Dor & Cor & $\begin{array}{c}\text { Temperatura } \\
\text { local }\end{array}$ & $\begin{array}{c}\text { Queixa } \\
\text { neural } \\
\text { associada }\end{array}$ & $\begin{array}{l}\text { Resposta } \\
\text { à } \\
\text { histamina }\end{array}$ & $\begin{array}{c}\text { Resposta } \\
\text { à } \\
\text { pilocarpina*** }^{* *}\end{array}$ \\
\hline 1 & mãos e pés $(D>E)^{\star \star \star \star}$ & $1 \mathrm{~m}$ & não & $\mathrm{N}$ & $\mathrm{N}$ & A & - & $A$ \\
\hline 2 & mãos e pernas (D>E) & $2 \mathrm{sem}$ & não & $\mathrm{N}$ & $\mathrm{N}$ & A & - & $\mathrm{P}$ \\
\hline 3 & perna $\mathrm{E}$ & $4 d$ & não & eritematosa & aumentada & A & - & $A$ \\
\hline 4 & braços e pernas & $6 \mathrm{~m}$ & não & eritematocianótica & $\mathrm{N}$ & A & completa & - \\
\hline 5 & pernas & $6 \mathrm{~m}$ & não & eritematosa & $\mathrm{N}$ & parestesia & - & $A$ \\
\hline 6 & pé $\mathrm{E}$ & $6 \mathrm{~m}$ & $\operatorname{sim}$ & cianótica & $\mathrm{N}$ & hiperestesia & incompleta & - \\
\hline 7 & mãos $(D>E)$ & $1 \mathrm{~m}$ & não & cianótica & $\mathrm{N}$ & parestesia & completa & - \\
\hline 8 & pernas $(E>D)$ & $3 \mathrm{~m}$ & não & cianótica & $\mathrm{N}$ & $A$ & completa & - \\
\hline 9 & pernas & $11 / 2 m$ & $\operatorname{sim}$ & eritematosa & aumentada & hiperestesia & incompleta & - \\
\hline 10 & pernas $(D>E)$ & $1 \mathrm{sem}$ & não & $\mathrm{N}$ & $\mathrm{N}$ & parestesia & - & $\mathrm{P}$ \\
\hline 11 & pernas $(D>E)$ & $1 \mathrm{~m}$ & não & $\mathrm{N}$ & $\mathrm{N}$ & A & incompleta & - \\
\hline
\end{tabular}

** Todos os pacientes apresentaram edema de consistência desprezível.

${ }^{* * *}$ Resposta à pilocarpina: sim = sudorese presente

**** $D=$ direito; $E=$ esquerdo

- Tempo de evolução: $d$ = dias, $m$ = mês, sem = semana

$\mathrm{N}=$ normal $; \mathrm{A}=$ ausente $\mathrm{P}=$ presente

Os testes de avaliação do sistema nervoso autônomo (teste de histamina = incompleta $\mathrm{e}$ pilocarpina = ausência de sudorese) estiveram alterados em 6 pacientes (Tabela 3).

Os pacientes foram acompanhados clinicamente, quanto à evolução do edema (Tabela 2), não recebendo nenhuma terapêutica específica para o mesmo, com exceção do paciente $\mathrm{n}^{\circ} 8$, que usou betametasona por conta própria e do paciente $n^{\circ} 9$, que durante internação hospitalar para investigação do edema recebeu prednisona por um mês por apresentar hiperestesia cutânea.
Os 5 pacientes do grupo A (Tabela 4), independente da terapêutica já vigente para 0 tratamento dos episódios reacionais (pentoxifilina, talidomida ou prednisona) tiveram melhora clínica importante (inclusive com remissão total em 3 pacientes) após a introdução (ou aumento da dosagem) da prednisona (de 20 a $80 \mathrm{mg}$ ).

Entre os 6 pacientes do grupo B (Tabela 2), 3 pacientes (nos 6, 10 e 11) evoluíram com a instalação de quadros reacionais $(2 \mathrm{com}$ neurite e 1 reação tipo I), após um período de 1 a 3 meses. Deste grupo B, 4 pacientes (os 3 que evoluíram com quadros reacionais e 1 paciente - no 9) com

Tabela 4 - Evolução clínica dos pacientes do Grupo A.

\begin{tabular}{|c|c|c|c|c|c|c|c|c|}
\hline Pacientes & Reação & Tempo & Tempo & Terapia & Terapia & Resp & línica & Tempo da \\
\hline $\mathrm{n}^{\circ}$ & & $\begin{array}{l}\text { evolução } \\
\text { do edema }\end{array}$ & $\begin{array}{l}\text { evolução } \\
\text { da reação }\end{array}$ & vigente* & associada ${ }^{\star *}$ & reação & edema & $\begin{array}{l}\text { resposta } \\
\text { clínica }\end{array}$ \\
\hline 1 & tipo I & $1 \mathrm{~m}$ & 1 sem. & $\begin{array}{c}\mathrm{PTX}^{*} 1 \\
(1200 \mathrm{mg})\end{array}$ & $\begin{array}{c}\text { pred } \\
(60 \mathrm{mg})\end{array}$ & melhora & melhora & $1 \mathrm{~m}$ \\
\hline 2 & tipo II & 2 sem & $3 \mathrm{~m}$ & $\begin{array}{c}\text { tal *2 }(200 \mathrm{mg}) \\
\text { pred}^{\star} 3(5 \mathrm{mg})\end{array}$ & $\begin{array}{c}\text { pred } \\
(30 \mathrm{mg})\end{array}$ & melhora & remissão & $1 \mathrm{~m}$ \\
\hline 3 & tipo I & $4 d$ & $2 \mathrm{~m}$ & - & $\begin{array}{c}\text { pred } \\
(80 \mathrm{mg})\end{array}$ & melhora & remissão & 2 sem. \\
\hline 4 & tipo I & $6 \mathrm{~m}$ & $1 \mathrm{sem}$ & - & $\begin{array}{c}\text { pred } \\
(60 \mathrm{mg})\end{array}$ & remissão & remissão & $1 \mathrm{~m}$ \\
\hline 5 & tipo I & $6 \mathrm{~m}$ & $1 \mathrm{~m}$ & $\begin{array}{c}\text { pred } \\
(5 \mathrm{mg})\end{array}$ & $\begin{array}{c}\text { pred } \\
(20 \mathrm{mg})\end{array}$ & melhora & melhora & $1 \mathrm{~m}$ \\
\hline
\end{tabular}


queixa neural associada de hiperestesia) fizeram uso de prednisona (20-60mg), com melhora clínica importante do edema (3 com remissão completa), após um período de 1 semana a 1 mês. Nos 2 pacientes restantes (nos 7 e 8 ) observou-se a remissão do edema no paciente que fez uso, por conta própria, de betametasona oral e, melhora do edema no outro, sem terapêutica associada (fazia uso apenas de amitriptilina, devido à parestesia).
A carga bacilar não parece ser um fator relevante na gênese dos edemas, uma vez que os pacientes apresentavam índices baciloscópicos (IB) diversos, com predomínio (7 pacientes) de $\mathrm{IB} \leq 1$ (sendo $2 \mathrm{com} \mathrm{IB}=0$ ); outros 4 pacientes apresentavam IB $\geq 3$ (sendo o maior igual a 3,66 ).

\section{DISCUSSÃO}

A ocorrência de edema associado à hanseníase foi objeto de estudo por alguns autores no passado9 2023 .

Alguns estudos 346 referem-se ao edema envolvendo face, mãos e pés como sendo um dos critérios clínicos para a caracterização da reação tipo I, especialmente em pacientes $\mathrm{BL}$.

Outros estudos analisando pacientes com reação tipo II, descreveram edema de mãos e/ou pés que foram interpretados como conseqüentes à obstrução transitória do fluxo linfático a nível dos linfonodos regionais 19 20. Nenhum dos pacientes de nossa casuística apresentou adenomegalias associadas às regiões acometidas pelo edema.

No presente estudo, observou-se a ocorrência de edema tanto em pacientes hansenianos que não apresentavam reações hansênicas clássicas, como em pacientes com reação. Entre estes a reação tipo I predominou, sendo que apenas um caso apresentou reação tipo II. Assim, a obstrução linfática transitória não parece explicar o aparecimento de edema em todos os pacientes.

Ommen9 relatou o acompanhamento de 6 pacientes com edema significativo dos membros inferiores, sendo 2 com edema também nas mãos, surgido após início da PQT, o que lhe pareceu estar associado à clofazimina, devido a uma possível obstrução linfática pelos seus cristais. Outros estudos foram feitos no intuito de se definir melhor esta correlação8 20. Este não parece ser também o fator determinante dos edemas, pois se a impregnação pela clofazimina fosse fator causal da obstrução linfática, seria de se esperar que este se mantivesse durante o tratamento PQT ou que se resolvesse com a suspensão da droga. Entre os pacientes por nós estudados, observou-se a ocorrência de edema tanto em pacientes em longo período de alta terapêutica pós-PQT (do $2^{\circ}$ ao $82^{\circ}$ mês), assim como em um paciente ainda virgem de terapêutica.

A observação de quadros de edema, precedendo a instalação de episódios reacionais (em 3 pacientes do grupo A e em 3 pacientes do grupo B) permite-nos especular ser o edema um pródomo ou um componente silencioso dos quadros reacionais clássicos. Um outro dado que reforça esta sugestão é a observação de regressão do edema em paciente do grupo B, que fez uso de prednisona (terapêutica usual dos quadros reacionais) e que não cursava com quadro reacional clássico (apenas hiperestesia, sem neurite).

Jopling et al2 postularam que o edema das pernas observado nos pacientes com hanseníase virchowiana seria resultante da combinação da gravidade com o aumento da permeabilidade capilar, sendo esta última, provavelmente, causada por uma combinação do comprometimento do endotélio capilar com o dano dos nervos que controlam a permeabilidade e o tônus capilar.

No presente estudo, os edemas se localizaram, preferencialmente, nos membros inferiores (em 10 dos 11 pacientes), reforçando a participação da ação da gravidade na localização dos mesmos.

Os testes de avaliação da integridade do sistema nervoso autônomo (testes de histamina e teste de pilocarpina) alterados em 6 pacientes confirma o comprometimento deste sistema na hanseníase, mesmo fora das lesões dermatológicas, visto que o mesmo foi realizado nas áreas com edema (sem lesão dermatológica aparente). O papel deste sistema na alteração do tônus vascular requer melhor esclarecimento.

O fato dos edemas serem todos depressíveis, apresentando em alguns casos, alterações associadas de temperatura e cor, reforçam a idéia de alterações na permeabilidade vascular na gênese destes edemas. O mecanismo etiopatogenético envolvido porém, nestas alterações precisa ser melhor avaliado.

Diversos estudos 1015161718 já demonstraram níveis elevados de TNF e IL-1, principalmente o TNF, no curso da hanseníase. Sarno et al17 
avaliando o possível papel do TNF e IL-1 nos estados reacionais observou no grupo controle (sem reação) concentrações elevadas em 02 pacientes, um com neurite e outro com edema importante, doloroso, no braço e mão esquerda, sugerindo que estes poderiam estar associados ao efeito local destas citocinas. Neste estudo, evidenciou-se níveis séricos elevados de TNF e IL- 1 nos estados reacionais em $50 \%$ dos pacientes (100\% dos pacientes com reação tipo I e $50 \%$ dos pacientes com reação tipo II) e concentrações de TNF maiores que $5.000 \mathrm{pg} / \mathrm{ml}$ observadas nos pacientes sem tratamento das reações.

O TNF, assim como a IL-1, são citocinas inflamatórias com ação sinérgica sobre o endotélio capilar7 1322 levando a um aumento na permeabilidade durante a reação inflamatória in vivo e ensaios in vitro 13 .

Okusawa et al7 sugeriram que a IL-1 seria o mediador do choque séptico e que o efeito do TNF nas células do endotélio vascular seria potencializado pela produção endógena de IL-1.

Wesselius et al22 induziram edema pulmonar em ratos, através da administração intratraqueal de TNF e IL-1 demonstrando o sinergismo de ação entre as duas.

A resposta clínica favorável do edema após a administração de prednisona oral em todos pacientes que apresentavam o edema associado à reação e naqueles que evoluíram com posteriores quadros reacionais clássicos sugere fortemente, um componente imunológico na etiopatogenia destes edemas.

As alterações de temperatura local e cor, assim como as queixas neurais associadas ao edema, poderiam ser também resultantes da ação de citocinas liberadas no curso da doença sobre os vasos e nervos, levando à formação do edema.

Considerando-se a possibilidade de uma gênese imunológica para o edema na hanseníase associado às reações clássicas hansênicas, poderíamos postular que medicações que atuem sobre os mediadores inflamatórios sejam atuantes nestes quadros.

O tratamento das reações, especialmente as formas crônicas persistentes é, sem dúvida, um dos maiores desafios na hanseníase. Tradicionalmente, utilizam-se esquemas padronizados de corticóide ou talidomida, entretanto deve-se admitir que cada paciente necessita de dosagens próprias para o controle dos diversos sintomas 21 .
Atalidomida é a droga de escolha, com efeito terapêutico excelente nas reações tipo II, porém com indicação limitada em mulheres em idade fértil pela elevada teratogenicidade. Sabe-se que os níveis de TNF- $\alpha$ estão relacionados com a reação inflamatória aguda da reação tipo II. A talidomida reduz a produção de TNF- $\alpha^{15}$ através do bloqueio do RNA mensageiro que comanda a produção desta citocina, o que poderia explicar o seu efeito no ENL5.

Contudo, não nos parece ser a talidomida uma opção para terapêutica do edema na hanseníase, visto que, quando associado a estados reacionais, o faz comumente em reações tipo I ou neurite.

Recentemente, há relatos que pentoxifilina pode ser uma opção em alguns casos de edema evitando o uso prolongado de corticóide, na dose plena de $1.200 \mathrm{mg} / \mathrm{dia}$. Aassociação com corticóide permitiria o uso de dose menores destes e encurtamento do tempo de tratamento. A pentoxifilina pode atuar favoravelmente como um fator reológico, em estados de aumento da coagulação sangüínea através da diminuição da adesividade e agregação plaquetária, aumento de antitrombina III, diminuição do fibrinogênio e outros efeitos. Também já se tem bem definido a ação no sistema imune em vários níveis: aumento da quimiotaxia e da deformidade leucocitária, diminuição da produção de TNF- $\alpha$ e da resposta leucocitária a IL-1 e TNF- $\alpha$, inibição da ativação linfocitária $T$ e $B$ e diminuição da atividade das células NK 1424.

O uso da pentoxifilina foi então tentado como terapia única na abordagem dos edemas não associados à reação (pacientes 8 e 10). Iniciouse com dose mínima (400mg) sendo programado aumento gradativo até a dose plena $(1200 \mathrm{mg})$.

Porém ao Iongo do $1^{\circ} \mathrm{m}$ ês de acompanhamento um dos pacientes (paciente 8) se automedicou com betametasona e o outro evoluiu com reação tipo I (paciente 10), sendo então medicado com prednisona (60mg).

Clinicamente, a abordagem diagnóstica dos quadros de edema nos pacientes hansênicos não é simples. Exige do profissional uma detalhada avaliação clínica e laboratorial, a fim de descartar as patologias clássicas associadas aos edemas (cardíacos, renais, hepáticos, nutricionais) que mesmo quando presentes não significam que seriam estas as causadoras do edema observado.

O presente estudo mostrou que os quadros de edema na hanseníase podem ocorrer como 
pródromos ou componentes de estados reacionais, mesmo na ausência de lesões dermatológicas. Esta observação pode ser inferida pela resposta terapêutica à pentoxifilina, talidomida e especialmente ao corticóide, o que sugere que mecanismos imunológicos estejam envolvidos na fisiopatologia destes edemas.

\section{REFERÊNCIAS BIBLIOGRÁFICAS}

1. Cochrane RG. Complicating conditions due to leprosy. In: Cochrane RG, Davey TF, McRobert G (eds) Leprosy in theory and practice. John Wright \& Sons Ltd, Bristol, p. 333, 1964.

2. Jopling $\mathrm{WH}$, McDougall AC. Manual de hanseníase $4^{\mathrm{a}}$ edição. Livraria Atheneu Editora, Rio de Janeiro, p. 26, 1991.

3. Joseph MS, Unilateral oedema of reversal reaction (RR) in bordeline leprosy. Leprosy Review 57:276-277, 1986.

4. Lienhardt C, Fine PEM. Type 1 reaction, neuritis and disability in leprosy. What is the current epidemiological situation? Leprosy Review 65:9-33, 1994.

5. Moreira AL, Sampaio EP, Zmuiozinas A, Frinpt $P$, Smith KA, Kaplan G. Thalidomide exerts its inhibitory action on tumor necrosis factor $\alpha$ by enhacing mRNA degration. Journal of Experimental Medicine 177:1675-1680, 1993

6. Naafs B. Treatment of Reactions and Nerve Damage. International Journal of Leprosy 64:521-528, 1996.

7. Okusawa S, Gelfand JA, Ikejima T, Connolly RJ, Dinarello CA. Interleukin 1 induces a shock-like state in rabbits:synergism with tumour necrosis factor and effect of cyclooxygenase inhibition. Journal of Clinical Investigation 81:1162-1672, 1988.

8. Oommen ST, Natu MV, Mahajan MK, Kadyan RS. Lymphangiographic evaluation of patients with clinical lepromatous leprosy on clofazimine. International Journal of Leprosy and Other Mycobacterial Diseases 62:32-36, 1994

9. Oommen T. Clofazimine-induced lymphoedema. Leprosy Review 61:289-296, 1990.

10. Parida SK, Grau GE, Zaheer AS, Mukherjee R. Serum tumor necrosis factor and interleukin 1 in leprosy and during lepra reactions. Clinical Immunology and Immunopathology 63:23-27, 1992.

11. Pfaltzgraff RE, Ramu G. Clinical leprosy. In: Hastings RC (ed) Leprosy. London, Churchill Livingstone, $2^{\mathrm{a}} \mathrm{ed}, \mathrm{p}$. 237-290, 1994.

12. Ridley DS, Jopling WH. Classification of leprosy according to immunity. A five group system. International Journal of Leprosy 34:255-273, 1966.
13. Royall JA, Berkow RL, Beckman JS, Cunningham K, Matalon S, Freeman BA. Tumor necrosis factor and interleukin $1 \alpha$ increase vascular endothelial permeability. American Journal of Physiology 257:1399-1410, 1989.

14. Samlaska CP, Winfield EA. Pentoxifilline. Journal of the American Academy of Dermatology 30:603-621, 1994.

15. Sampaio EP, Sarno EN, Galilly R, Cohn ZA, Kaplan G. Thalidomide selectively inhibits tumor necrosis factor $\alpha$ production by stimulated human monocytes. Journal of Experimental Medicine 173:699-703, 1991.

16. Santos DO, Suffys PN, Bonifácio K, Marques, MA, Sarno $\mathrm{EN}$. In vitro tumor necrosis factor prodution by mononuclear cells from lepromatous leprosy patients and from patients with Erythema Nodosum Leprosum. Clinical Immunology and Immunopathology 67:199-203, 1993.

17. Sarno EN, Grau GE, Vieira LMM, Nery JAC. Serum levels of tumour necrosis factor-alpha and interleucin- $1 \beta$ during leprosy reactional states. Clinical and Experimental Immunology 84:103-108, 1991.

18. Silva CL, Foss NT, Tumor necrosis factor in leprosy patients. Journal of Infectious Diseasis 159:787-790, 1989.

19. Stanley JNA, Pearson JMH, Ridley DB. Episodic edema in type 2 lepra reaction can be caused by transient lymphatic obstruction in the lymphonode. International Journal of Leprosy 54:231-235, 1986.

20. Tyagi PY, Oommen T. Pedal edema following clofazimine therapy; a case report. International Journal of Leprosy and Other Mycobacterial Diseases 61:636, 1993.

21. Waters MFR. Treatment of reactions in leprosy. Leprosy Review 45:337-341, 1974.

22. Wesselius LJ, Smirnoy IM, O'Brien-Lardner AR, Nelson ME. Synergism of intra tracheally administered tumor necrosis factor with interleukin 1 in induction of lung edema in rats. Journal of Laboratory and Clinical Medicine 125:618-625, 1995

23. Wheate HW. Acute edema in leprosy. International Journal of Leprosy 30:387-394, 1962.

24. Zabel P, Schade FU, Schlaak M. Inhibition of endogenous TNF formation by pentoxifiline. Immunobiology 187:447, 1993. 